\title{
On the Extreme Positive Feedback Star-Forming Mode from Massive and Compact Superstar Clusters
}

\author{
Guillermo Tenorio-Tagle, Sergiy Silich, Ary Rodríguez González \\ Instituto Nacional de Astrofísica Optica y Electrónica, AP 51, 72000 Puebla, México; \\ gtt@inaoep.mx \\ and \\ Casiana Muñoz-Tuñón \\ Instituto de Astrofísica de Canarias, E 38200 La Laguna, Tenerife, Spain; cmt@ll.iac.es
}

\begin{abstract}
The force of gravity acting within the volume occupied by young, compact and massive superstar clusters, is here shown to drive in situ all the matter deposited by winds and supernovae into several generations of star formation. These events are promoted by radiative cooling which drains the thermal energy of the ejected gas causing its accumulation to then rapidly exceed the gravitational instability criterion. A detailed account of the integrated ionizing radiation and mechanical luminosity as a function of time is here shown to lead to a new stationary solution. In this, the mass deposition rate $\dot{M}$, instead of causing a wind as in the adiabatic solution, turns into a positive feedback star-forming mode equal to the star formation rate. Some of the implications of this extreme positive feedback mode are discussed.
\end{abstract}

Subject headings: HII regions: clusters: winds - galaxies

\section{Introduction}

The discovery by HST and large ground-based telescopes, of a large population of superstar clusters (SSCs) in a large variety of galaxies (see Ho 1997, Martín Hernández et al. 2005, and Melo et al. 2005), has driven the community to speculate about the impact that these new units of star formation may have on the ISM of their host galaxies. SSCs with a mass, $\mathrm{M}_{S C}$, ranging from a few $\times 10^{5} \mathrm{M}_{\odot}$ to up to $6 \times 10^{7} \mathrm{M}_{\odot}$ (see Walcher et al. 2004; Pasquali et al. 2004) all within a small volume of radius 3 to $10 \mathrm{pc}$, are indeed some of 
the most energetic entities within galaxies. Their large UV photon output and mechanical energy are now believed to be the largest negative feedback agents in starburst galaxies, leading not only to a large-scale structuring of the ISM and to limit star formation, but also to be the agents capable of establishing as in M82 a supergalactic wind, thereby removing processed material from galaxies and causing the contamination of the IGM (Tenorio-Tagle et al. 2003).

In the present series of papers however, we have shown that there is a threshold limit that massive and energetic compact clusters may cross to find out that radiative cooling inhibits their stationary outflow condition (Silich et al., 2004) and then the matter ejected by the stellar sources, unable to escape, is to accumulate within the star cluster volume (Tenorio-Tagle et al. 2005). The facts in such cases are that radiative cooling drastically diminishes the sound speed and the pressure gradient within the thermalized ejected matter, inhibiting the possibility of a wind. Radiative cooling upsets the stationary condition in which the deposited matter $(\dot{M})$ has to equal, at all times, the amount of matter streaming out of the SC volume, $\dot{M}=2 \mathcal{L}_{S C} / v_{\infty}^{2}=4 \pi R_{S C}^{2} \rho_{S C} c_{S C}$, where $\mathcal{L}_{S C}$ is the amount of energy injected by stellar winds and $\mathrm{SNe}, c_{S C}$ is the local sound speed at the SC surface and $v_{\infty}$ is the resultant wind terminal speed in the absence of radiative cooling. As soon as this happens, the ejecta begins to accumulate, promoting larger densities and an even faster cooling within the SSC volume.

The location of the threshold line in the $\mathcal{L}_{S C}$ vs size $\left(R_{S C}\right)$ diagram (see Figure 1), the line that defines whether or not a wind is inhibited, depends on several variables. It depends on the size of the star-forming region $\left(R_{S C}\right)$, the metallicity of the ejected gas, which has a strong impact on the cooling curve (see paper III). It also depends on the assumed $\mathcal{L}_{S C} / \dot{M}$ or adiabatic terminal speed $\left(v_{\infty}\right)$ of the wind. The latter is also bound to the usual assumption that the energy deposited by SN is always $10^{51} \mathrm{erg}$ but the mass of potential SNe within the cluster ranges from, say, $100 \mathrm{M}_{\odot}$ to $8 \mathrm{M}_{\odot}$ and so the injection speed (similar to $v_{\infty}$ ) and the deposited amount of matter are also functions of time. Another factor that strongly affects the location of the threshold line is the thermalization efficiency $(\epsilon)$ which simply defines the fraction of the mechanical energy that can be evenly spread within the cluster volume. Estimates of $\epsilon$ by several authors lead to values between 1 (Chevalier \& Clegg; 1985) to 0.03 (see Melioli \& Del Pino 2004 and references therein) and depends simply on the proximity of the sources undergoing winds and $\mathrm{SNe}$, which through radiation may reduce the amount of energy available after thermalization. We have shown that there are three different types of solutions: SSCs far away from the threshold line (low mass, low energy clusters) undergo a quasi-adiabatic evolution well described by the Chevalier \& Clegg (1985) and Cantó et al., (2000) solution. More energetic clusters are to have strongly radiative winds. Cooling hardly affects their velocity $\left(v_{w} \sim v_{\infty}\right)$ and density distribution $\left(\rho_{w} \propto r^{-2}\right)$, but their temperature 
instead of falling as $r^{-4 / 3}$, it falls rapidly to $T_{w} \sim 10^{4} \mathrm{~K}$ close to the SC boundary and the more so, the closer they are to the threshold line. The strongly radiative winds around such clusters lead, compared to the adiabatic solution, to very much reduced X-ray envelope sizes. The third solution is for clusters above the threshold line. These would have their winds inhibited and as shown below, this turns them into very efficient positive feedback star-forming agents.

Here we follow the evolution of a massive and compact cluster and show the fate of the mass reinserted back, through winds and SNe, into the SC volume. Section 2 and 3 give a full description of SSC as positive feedback agents and section 4 summarizes our conclusions.

\section{Feedback from massive and compact SSC}

Here we follow the evolution of a stellar cluster, assumed to be the result of an instantaneous or coeval burst of star formation. The cluster has an initial total mass in stars $\left(M_{S C}\right)$ equal to $10^{7} \mathrm{M}_{\odot}$ within an $R_{S C}=5 \mathrm{pc}$. We assume a standard Salpeter IMF and an upper and lower mass limits equal to $100 \mathrm{M}_{\odot}$ and $1 \mathrm{M}_{\odot}$, respectively. The mechanical energy of the cluster $\mathcal{L}_{S C} \sim 10^{41} \mathrm{erg} \mathrm{s}^{-1}$ places our example cluster in the zone where stationary superwinds are inhibited (see Figure 1) and thus the mass returned by the stars $(\dot{M})$ begins immediately to accumulate within the star cluster volume, favoring an even faster cooling. Figure 2 shows how the density of the accumulating gas $\left(\rho_{a c}=3 \dot{M} t / 4 \pi R_{S C}^{3}\right.$; where $t$ the evolution time), grows as a function of time within the SSC volume.

Following this trend, clearly there is a moment when the density of the accumulating gas will exceed the gravitational instability criteria

$$
\rho_{J}=\frac{\pi \gamma k}{4 G \mu} T R_{s c}^{-2} \sim 2.3 \times 10^{-20}\left(\frac{T}{100 K}\right)\left(R_{S C 1}\right)^{-2} \mathrm{gcm}^{-3}
$$

where $\rho_{J}$ is the Jeans density and $k, G, \mu$ and $\gamma$ are the Boltzmann constant, gravitational constant, mean mass per particle and the ratio of specific heats $(5 / 3)$, respectively and $R_{S C 1}$ is the SSC radius in pc units. At that moment, when $\rho_{a c}=\rho_{J}$ collapse will inevitably proceed.

The initial ample supply of UV photons $\left(N^{0}=10^{54}\right.$ photons $\mathrm{s}^{-1}$, see Figure 3a), exceeds at first the number of recombinations within the volume occupied by the reinserted gas and the resultant HII region, given the large metallicities of the ejecta, is here assumed to rapidly approach an equilibrium temperature $T_{H I I} \sim$ a few $10^{3} \mathrm{~K}$. At these temperatures the sound speed $\left(<10 \mathrm{~km} \mathrm{~s}^{-1}\right)$ remains well below the escape speed $\left(V_{\text {esc }}=\left(2 G M_{S C} / R_{S}\right)^{1 / 2} \sim 130\right.$ $\mathrm{km} \mathrm{s}^{-1}$ ) and the reinserted gas would inevitably continue to accumulate to rapidly (within 
$1.5 \times 10^{6} \mathrm{yr}$ ) reach the value of the Jeans density for a gas at say, $T_{H I I}=5000 \mathrm{~K}$, and collapse into a new stellar generation. The event gives rise to a new phase of matter accumulation, which once more will rapidly approach $\rho_{J}$ (for $T=5000 \mathrm{~K}$ ) and undergo collapse within a free-fall time, of the order of $10^{5} \mathrm{yr}$, while transforming $\sim 2 \times 10^{5} \mathrm{M}_{\odot}$ into stars. All stellar generations resultant from mass accumulation within the SSC volume have here been assumed to also acquire a Salpeter IMF with similar upper and lower mass limits as those imposed to the main superstar cluster, and their resultant properties (mechanical energy and UV photon output) have been added to those produced by the main cluster.

A few, almost identical, stellar generations are expected from the accumulation process (solid rising lines in Figure 2), every time that the accumulated gas density $\rho_{a c}$ reaches $\rho_{J}(5000 K)$ (dashed line in Figure 2). The situation changes slightly when the number of ionizing photons $\left(N^{0}\right)$, despite the added contribution of secondary stellar generations, becomes insufficient to fully ionize the accumulated matter within the star cluster volume. This is due to the evolution of the main cluster, whose UV photon output begins to fall as $t^{-5}$ after $\sim 3.5 \mathrm{Myr}$ (dotted line in Figure 3a). Figure 2 shows $\rho_{H I I}$ (thin solid line), the maximum density within the accumulating volume that can be supported fully ionized by the UV radiation produced by the evolving cluster $\left(\rho_{H I I}=\left(3 N^{0} \mu^{2} / 4 \pi R_{S C}^{3} \beta\right)^{1 / 2}\right.$; where $\mu=1.4 m_{H}$ and $\beta$, the recombination coefficient to all levels above the ground level $=2.59$ $\left.\times 10^{-13} \mathrm{~cm}^{-3} \mathrm{~s}^{-1}\right)$. During the accumulation process, once $\rho_{a c}$ exceeds $\rho_{H I I}$, the ionized volume begins to shrink to end up as a collection of ultra compact HII regions around the most massive stars left within the cluster, while the bulk of the ejected material, now recombined, continues to cool, approaching rapidly (within a time-scale of less than 1000 yr) a temperature $\sim 100 \mathrm{~K}$. Matter is at all times uniformly replenished within the whole SSC volume, and thus the gas density presents an almost uniform value. However, the accumulating gas now has two different temperatures $\left(T_{H I I}\right.$ and $\left.100 \mathrm{~K}\right)$ and as $\rho_{a c}$ grows and the fraction of the ionized volume $\left(f_{H I I}=3 N^{0} \mu^{2} / 4 \pi R_{S C}^{3} \beta \rho_{a c}^{2}\right)$ shrinks, the size of cold condensations (at $100 \mathrm{~K}$ ) able to become gravitationally unstable and their free-fall time also become smaller.

The drop in the number of ionizing photons (Figure 3a) and the consequent growth of the neutral volume (Figure $3 \mathrm{~b}$ ) lead then to a second important condition in which the characteristic accumulation time $\tau_{a c}=\frac{4}{3} \pi \rho_{g a s} R_{S C}^{3}\left(1-f_{H I I}\right) / \dot{M}$ becomes equal to the freefall time $t_{f f}=\left(3 \pi / 32 G \rho_{\text {gas }}\right)^{1 / 2}$. This condition defines $\rho_{\text {gas }}$, the density above the Jeans instability limit for a neutral condensation at $100 \mathrm{~K}\left(\rho_{J}(100 \mathrm{~K})\right)$ :

$$
\rho_{\text {gas }}=\left[\frac{27 \dot{M}^{2}}{512 \pi G\left(1-f_{H I I}\right)^{2}}\right]^{1 / 3} R_{S C}^{-2}=\frac{1.15 \times 10^{-18}}{\left(1-f_{H I I}\right)^{2 / 3}}\left(\frac{\dot{M}}{1 M_{\odot} y r^{-1}}\right)^{2 / 3}\left(\frac{R_{S C}}{1 p c}\right)^{-2},
$$

and thus once $\rho_{a c}$ (see Figure 2) becomes equal to $\rho_{\text {gas }}$, a new stationary solution becomes 
possible.

Everything happens very rapidly, compared to the evolution time-scale of the parental cluster $(\sim 40 \mathrm{Myr})$, and almost at the same time. The ejected matter is thermalized within the SSC volume and immediately begins to cool. At the same time that it accumulates making cooling even faster. This allows it to rapidly reach the required $\rho_{\text {gas }}$ value, above the Jeans instability limit, that warrants its collapse in a similar time-scale, while the collapsing material is replenished by the newly ejected matter. When this happens, the mass deposition rate from the cluster becomes equal to the rate of star formation. Gravitational collapse and star formation within the star cluster volume and with the matter injected by all sources, drive in this way a new stationary condition through a new era of quasi-continuous star formation in which $\dot{M}$ is equal now to the star formation rate (SFR).

\section{The quasi-continuous star formation era}

All new generations of stars are due to deposit their ejecta from winds and SNe during $\approx 40 \mathrm{Myr}$, the expected duration of the type II SN phase in coeval star clusters (Leitherer \& Heckman 1995), and at a rate that depends only on the mass of the stellar generation. Re-processing leads to an escalating $\dot{M}$, as this is directly proportional to the total mass in stars

$$
M_{\text {stars }}(t)=M_{s c}+\int_{t_{C}}^{t} S F R(t) \mathrm{d} t
$$

created within the star cluster volume; where $t_{C}$ marks the start of the quasi-continuous star-forming phase and $M_{S C}$ accounts for the initial mass in stars and for all generations prior to the quasi-continuous star formation phase (see Figure 2). This promotes a larger star formation rate, and a faster fulfillment of the collapse conditions $\left(\rho_{g a s} \geq \rho_{J}\right.$, and $\left.\tau_{a c}=t_{f f}\right)$. Under these conditions the SFR is:

$$
S F R(t)=\frac{\mathrm{d} M_{\text {stars }}}{\mathrm{d} t}=\delta M_{\text {stars }}(t)
$$

This equation has an exponential solution:

$$
M_{\text {stars }}(t)=M_{s c} \exp \left[\left(t-t_{C}\right) / \tau\right]
$$

where the characteristic time $\tau=\delta^{-1}$. Equation (5) remains valid during the supernovae phase of the parental cluster. Within this time interval, the continuous re-processing of the ejected material drives, as shown in Figure 4, the star formation rate to grow exponentially:

$$
S F R(t)=\frac{\mathrm{d} M_{\text {stars }}}{\mathrm{d} t}=\frac{M_{s c}}{\tau} \exp \left[\left(t-t_{C}\right) / \tau\right] .
$$


Note that the characteristic time $\tau$ is defined by the coeval star cluster model and is applicable to both the initial cluster and also to every new generation of stars formed from the re-ejected material. If one assumes that the energy and mass deposition rates remain constant during a star cluster life time and that $v_{\infty}$, the resultant terminal speed in the absence of radiative cooling, equals $1500 \mathrm{~km} \mathrm{~s}^{-1}$ for all clusters, upon normalizing to the energy deposition rate adopted for the parental $10^{7} \mathrm{M}_{\odot} \operatorname{cluster}\left(\mathcal{L}_{i}=10^{41} M_{i} / 10^{7} M_{\odot}\right.$ erg $\left.\mathrm{s}^{-1}\right)$, then $\tau=M_{i} / \dot{M}_{i}=M_{i} v_{\infty}^{2} /\left(2 \mathcal{L}_{i}\right)=7.5 \times 10^{7} \mathrm{yr}$, where $M_{i}$ is the mass in each stellar generation, including the initial or parent cluster. The total mass in stars born within the star cluster volume, over the characteristic time $\tau \approx 40 \mathrm{Myr}$, is given by equation (5).

\section{Conclusions}

We have here shown that massive and compact coeval clusters instead of driving a superwind able to disperse the surrounding ISM and even channel its way into the IGM, events that have make them been regarded as negative feedback agents, they are in fact extreme examples of positive star formation feedback.

Massive and compact coeval clusters appear in the $\mathcal{L}_{S C}$ vs size diagram above the threshold line, in the region where radiative cooling inhibits the development of stationary superwinds. We have here shown that in such cases the matter reinserted, through stellar winds and supernovae, is unable to escape and that after a short phase of matter accumulation, a new stationary solution in which $\dot{M}$ becomes equal to the SFR is rapidly met. A positive feedback condition in which new stellar generations result in situ, from the collapse of the matter reinserted by the sources evolving within the star cluster volume.

The secondary star formation process while causing a faster mass deposition rate, drives the SFR to grow from 0.1 to $0.25 \mathrm{M}_{\odot} \mathrm{yr}^{-1}$ over the parent cluster supernova phase $(\sim 40$ Myr). The continuous re-processing of the ejected material leads effectively to a continuous transformation of the high mass stars into a low mass $\left(\leq 8 \mathrm{M}_{\odot}\right)$ population, keeping constant the total mass of the stellar component. In this way massive clusters may survive as gravitationally bound systems (see discussion in Dale et al. 2005 and Martín-Hernández et al. 2005 and references therein).

A central issue, regarding ISM studies, is the fact that the more massive and compact clusters (as those detected by HST and large ground-based telescopes), are unable to generate superwinds and shed their metals into the ISM or the IGM. Their evolution leads to many stellar generations and thus to a mixture of stellar populations, all contaminated by the products from former stellar generations. Exacerbated episodes of star formation that leave 
no trace of their evolution in the ISM. Clearly, if the most massive star formation events do not contribute to the chemical evolution of their parent galaxy, then several issues, such as the mass-metallicity relationship between galaxies, ought to be revised.

We thank our anonymous referee for comments and suggestions. This study has been supported by CONACYT - México, research grant 47534-F and AYA2004-08260-CO3-O1 from the Spanish Consejo Superior de Investigaciones Científicas.

\section{REFERENCES}

Cantó, J., Raga, A.C. \& Rodriguez, L.F. 2000, ApJ, 536, 896

Chevalier, R.A. \& Clegg, A.W. 1985, Nature, 317, 44

Dale, J.E., Bonnell, I.A., Clarke, C.J. \& Bate, M.R. 2005, MNRAS, 358, 291

Ho, L. C. 1997, Rev.MexAA, Conf. Ser. 6, 5

Leitherer, C. \& Heckman, T.M., 1995, ApJS, 96, 9

Martín-Hernández, N.L., Schaerer, D. \& Sauvage, M. 2005, A\&A 429, 449

Melioli, C. \& de Gouveia Del Pino E. M. 2004 A\&A 424, 817

Melo, V. P., Muñoz-Tuñón, C., Maíz-Apellãniz, J. \& Tenorio-Tagle, G. 2005, ApJ, 619, 270

Pasquali, A., Gallagher, J. S. \& de Grijs, R. 2004 A\&A 415, 103

Silich, S., Tenorio-Tagle G. \& Rodríguez González, A. 2004, ApJ, 610, 226 (Paper II)

Tenorio-Tagle, G., Silich, S. \& Muñoz-Tuñón, C. 2003, ApJ, 597, 279

Tenorio-Tagle, G., Silich, S., Rodríguez-González A. \& Muñoz-Tuñon, C., 2005, ApJ 620, 217, (Paper III)

Walcher, C.J., van der Marel, R.P., McLaughlin, D., Rix, H.-W., Böker, T., Häring, N., Ho, L.C., Sarzi, M. \& Shields, J.C. 2005, ApJ, 618, 237 
Fig. 1. - The threshold line. The location of the threshold line, the line that divides the $\mathcal{L}_{S C}$ vs size $\left(R_{S C}\right)$ diagram into two distinct areas. There the matter deposited within the SSC volume, through winds and $\mathrm{SNe}$, ends up either streaming away as a stationary (adiabatic or radiative) wind (below the line) or it accumulates to end up steadily being driven into new episodes of star formation (above the line). Two different locations of the threshold line, for an assumed full thermalization efficiency $(\epsilon=1)$, are display for an ejecta metallicity value equal to solar and ten times solar. The change in location for different values of $\epsilon$ is also indicated.

Fig. 2.- Positive star-forming feedback. The figure shows the rapid growth of the reinserted gas density within the SSC volume (rising solid lines), interrupted as it reaches the gravitational instability criterium $\left(\rho_{J}\right)$, leading to a new stellar generation and to a new phase of matter accumulation. The situation changes slightly as the available photon flux becomes unable to sustain all the SSC volume fully ionized, allowing for recombination and further cooling of the recombined matter, and thus to a much lower limit of the Jeans instability criterium. This allows the accumulation time to become comparable to the free-fall time, establishing a new stationary condition in which $\dot{M}=$ SFR. The density value $\left(\rho_{\text {gas }}\right)$ required for this condition is also indicated in the figure as well as the maximum density value $\left(\rho_{H I I}\right)$ that can be supported fully ionized within the SSC volume, with the available UV photon flux.

Fig. 3.- Figure (a) show the evolution of the UV photon flux produced by the main star cluster (dotted line) and the total flux resultant from the contributions from all further stellar generations (solid line). b) displays $f_{H I I}$, the fraction of the SSC volume that can be kept fully ionized with the available UV stellar photon output.

Fig. 4.- Positive star-forming feedback. The evolution of the star formation rate throughout the life-time of the main cluster. 


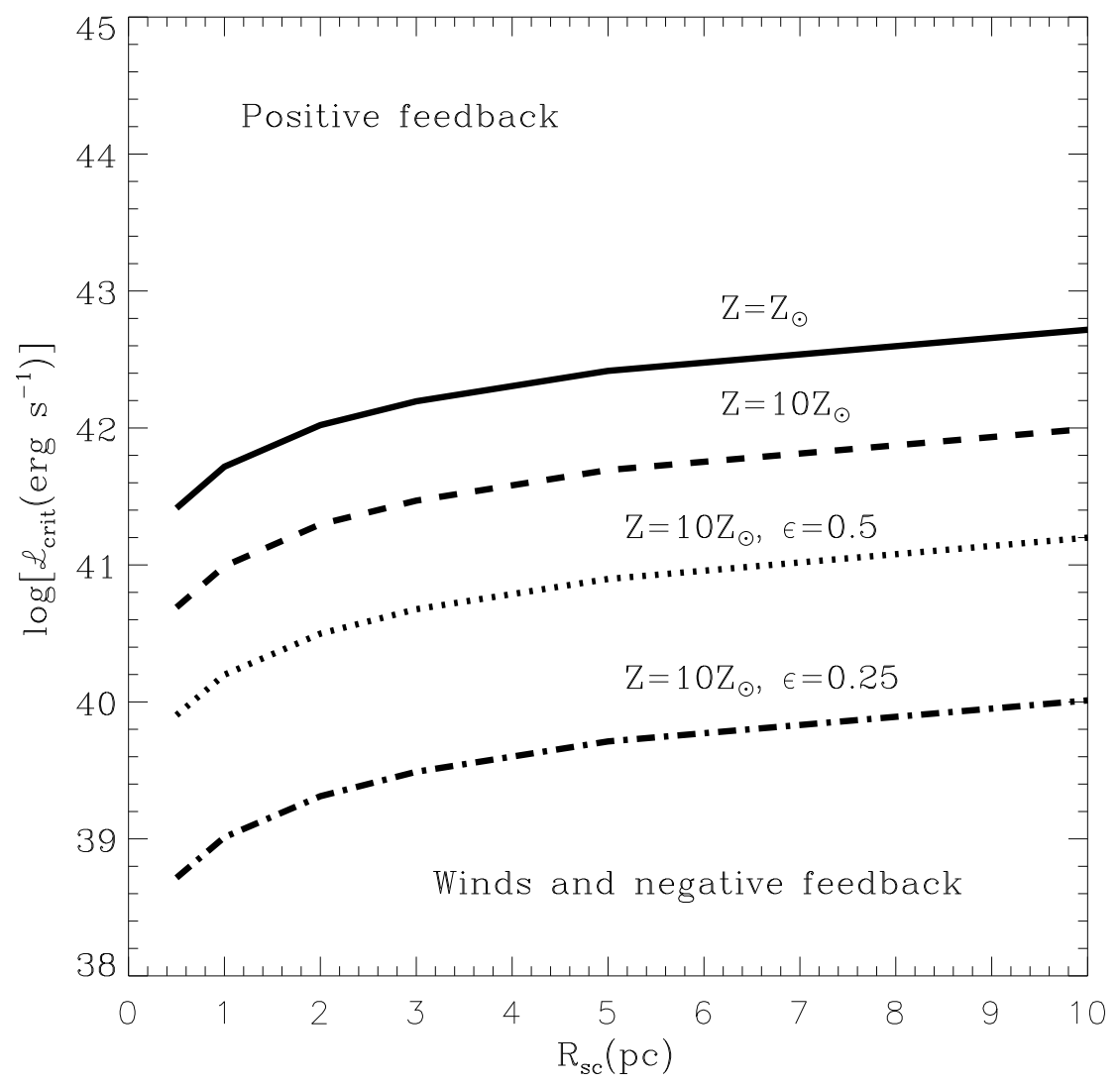




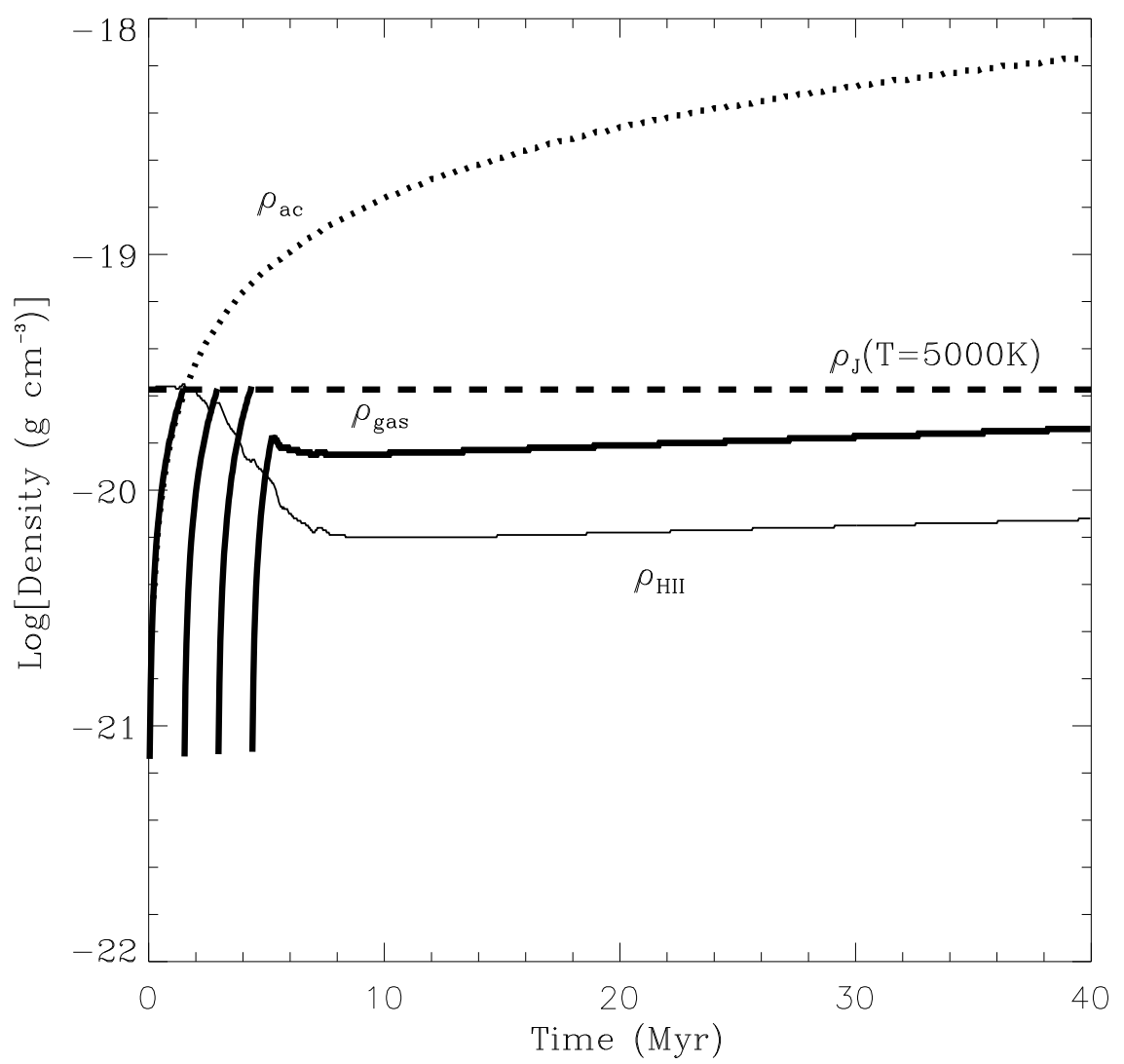



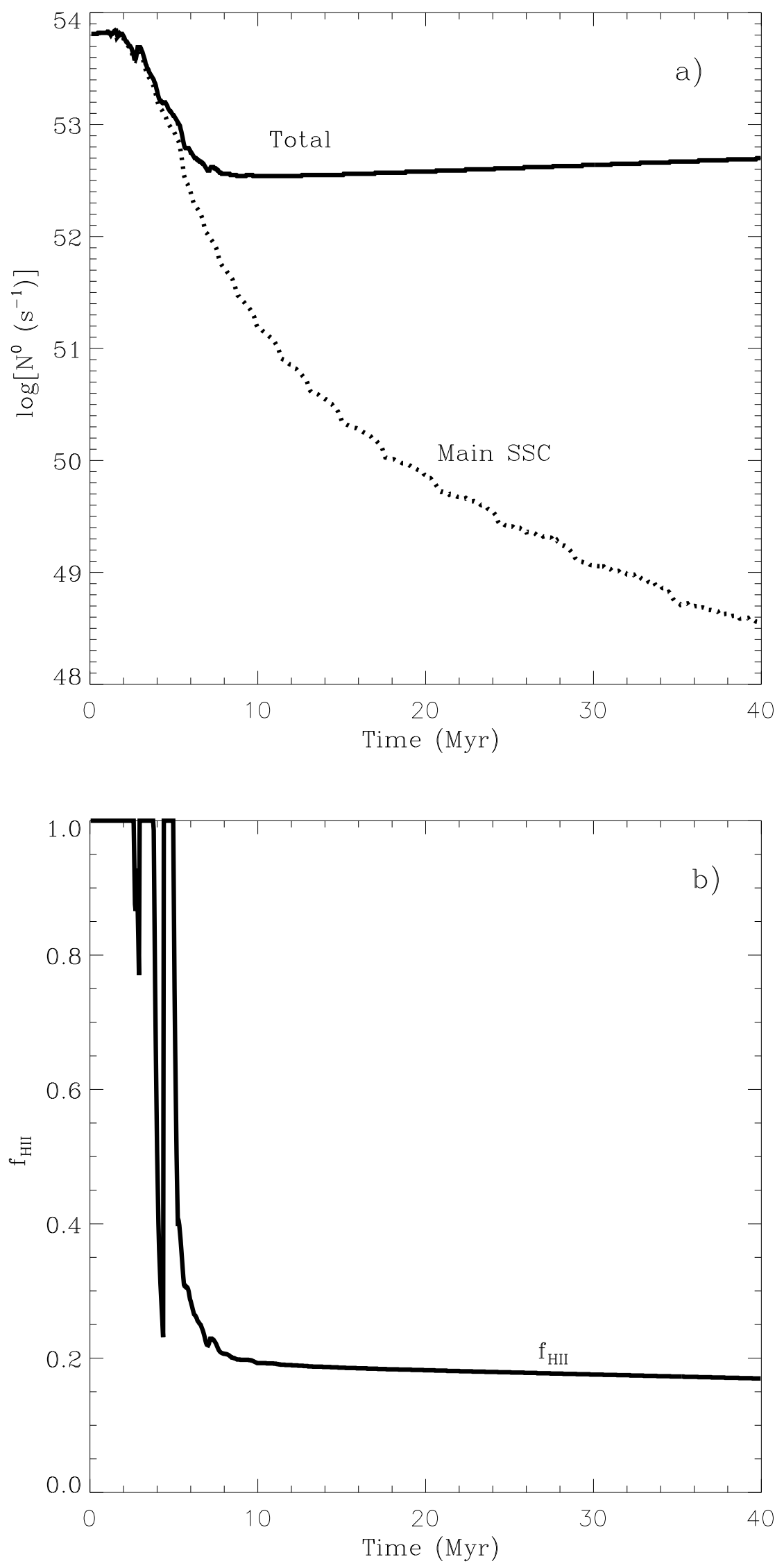


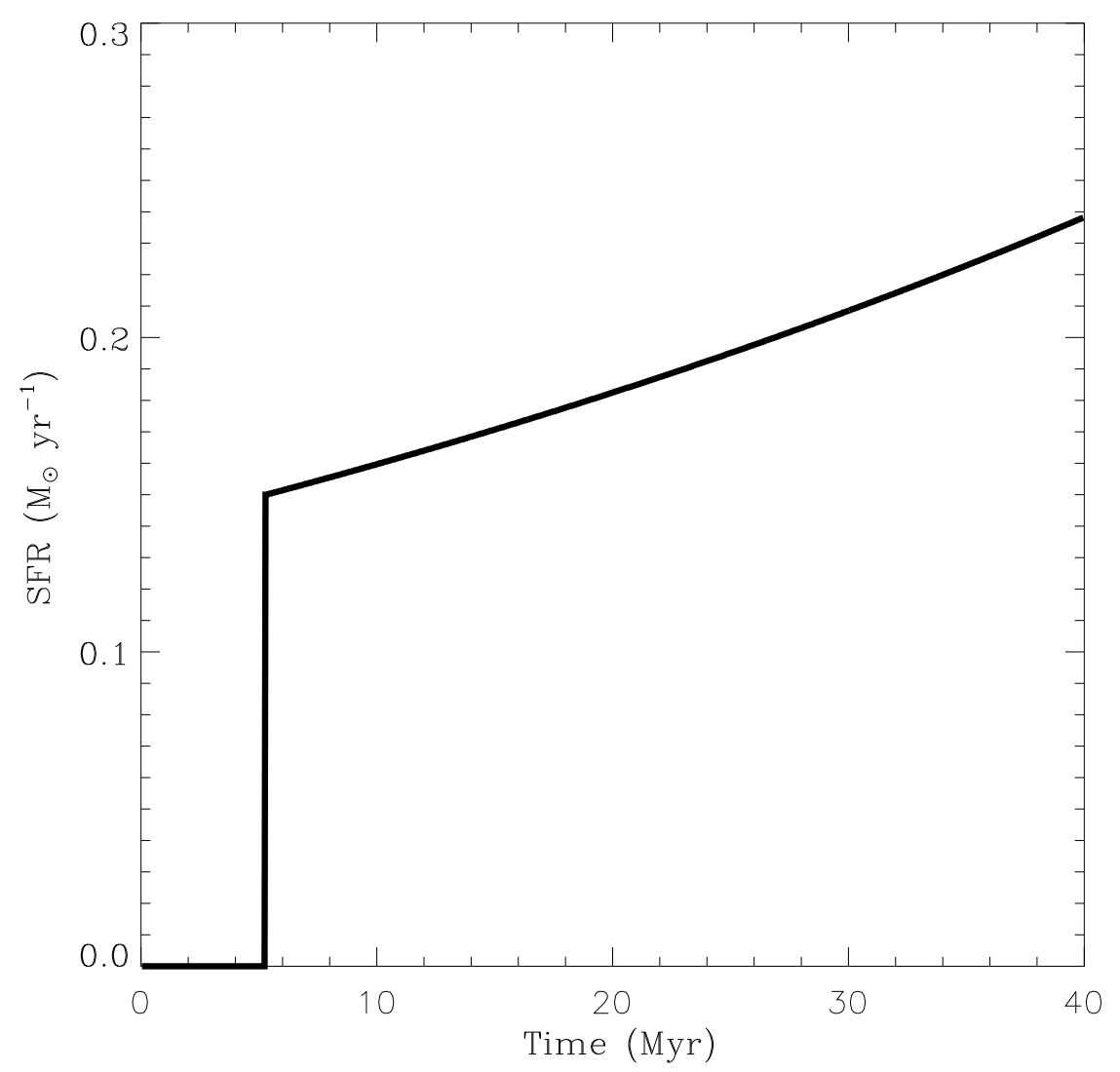

